\title{
Envejecimiento y finanzas: un modelo de comportamiento a largo plazo en España*
}

\author{
Aging and personal finance: a long-term behavior model of the Spanish population
}

\author{
Montserrat Díaz-Fernández \\ Doctora en Economía Cuantitativa, Universidad de Oviedo, \\ Oviedo-España,mdiaz@uniovi.es \\ María del Mar Llorente-Marrón \\ Doctora en Economía Cuantitativa, Universidad de Oviedo, \\ Oviedo-España, mmarron@uniovi.es \\ María Paz Méndez-Rodríguez \\ Doctora en Economía Cuantitativa, Universidad de Oviedo, \\ Oviedo-España, mpmendez@uniovi.es
}

\begin{abstract}
Cómo citar / How to cite
Díaz-Fernández, M., Llorente-Marrón, M. del M. \& Méndez-Rodríguez, M. P. (2019). Envejecimiento y finanzas: un modelo de comportamiento a largo plazo en España. Revista CEA, 5(10), 35-48. https://doi.org/10.22430/24223182.1442
\end{abstract}

Recibido: 12 de enero de 2019

Aceptado: 3 de abril de 2019

\section{Resumen}

El envejecimiento demográfico determina las características del ámbito económico. La hipótesis de estabilidad en el consumo del ciclo de vida del individuo hace del envejecimiento demográfico un factor que podría afectar al nivel de ahorro agregado. Normalmente, en la modelización del comportamiento de los activos financieros, son los factores de coyuntura económica los tenidos en cuenta quedando el foco demográfico relegado a un segundo plano. Diversos estudios empíricos han encontrado una elevada correlación entre la distribución por edades y las cotizaciones bursátiles. Con base en lo anterior, este trabajo realiza un ejercicio empírico con objeto de captar la conexión entre el papel que las finanzas personales ocupan en el ciclo de vida del individuo y el contexto demográfico. Mediante la especificación de un Modelo de Corrección del Error (MCE) se realiza un análisis de cointegración, que visualiza la vinculación estable a largo plazo entre el consumo de productos financieros y los cambios en la estructura por edades de la población.

Palabras clave: envejecimiento demográfico, finanzas personales, análisis de cointegración.

\footnotetext{
${ }^{*}$ Este artículo se deriva del proyecto titulado «Envejecimiento y finanzas: un modelo de comportamiento a largo plazo en España» enmarcada en la línea de modelización econométrica del fenómeno económico y demográfico. El proyecto ha sido financiado con recursos propios.
} 


\begin{abstract}
The aging of a population determines the characteristics of its economic environment and, according to the life-cycle hypothesis, it may affect the level of aggregate saving. Generally, the modeling of financial asset behavior considers factors of economic events and neglects the demographic approach. However, several empirical studies have found a high correlation between age distribution and stock prices. Based on those ideas, this empirical work aims at capturing the connection between the role that personal finance plays in individuals' lifecycles and their demographic context. By means of an Error Correction Model (ECM), a cointegration analysis was conducted to visualize the stable, long-term relationship between the consumption of financial products and changes in the age structure of the population.
\end{abstract}

Keywords: Population aging, personal finance, cointegration analysis.

\title{
1. INTRODUCCIÓN
}

La identificación y diagnóstico del denominado problema de la población ha evolucionado. Saber si en un momento y ámbito territorial concreto hay, o no, exceso de población constituye una cuestión claramente discutible. Actualmente es, quizá, el envejecimiento demográfico, el aspecto que más alarma suscita en el mundo desarrollado (Cabré i Pla \& Pérez Díaz, 1996; Pérez, 2005; Díaz-Fernández, 2018).

Los cambios en la estructura por edades de la población condicionan las características de la esfera económica. Cada cohorte demográfica incorpora aspectos diferentes en términos de estructura de preferencias, necesidades y también capacidad productiva. A nivel agregado, la incorporación generalizada de efectivos poblacionales a las cohortes de edad adulta modifica el peso que en el conjunto de la población presentan los grupos de edad avanzada y repercute necesariamente en el ámbito económico. El alcance de dichos cambios se vincula tanto a la generación de riqueza, producción y consumo como al ahorro y formación de activos financieros. Desde una perspectiva demográfica, primer y segundo dividendo demográfico se verían consecuentemente afectados (Roa García, 2008; Pinto, 2016).

A lo largo del ciclo de vida los perfiles de renta, consumo y ahorro de los individuos cambian. La teoría del ciclo de vida desarrollada por Modigliani (1986) ha sido de gran ayuda para entender la importante relación que existe entre el consumo y el ahorro de la población activa en relación con las personas que ya se han jubilado. Dicha teoría ha contribuido a despertar en la sociedad la preocupación por el bienestar económico futuro. Los individuos ahorran durante su periodo laboral, materializando un gasto inferior al que su poder adquisitivo podría permitir y pensando que, en la etapa de jubilación, los ingresos serán inferiores a los gastos. Los individuos se endeudan fundamentalmente cuando son jóvenes para la adquisición de vivienda, ahorran en la edad adulta para el pago de la deuda y en previsión de la vejez y gastan en la jubilación (Paxson, 1996; Deaton, 1997). Empíricamente, distintos estudios señalan la edad de cambio en las pautas de consumo, así como la coexistencia de procesos de acumulación y desacumulación de activos ahorrados en el pasado. A partir de los 45 años de edad, los consumidores estabilizan y ralentizan sus pautas de consumo a la vez que materializan tasas de ahorro positivas (Gourinchas \& Parker, 2001; Carroll \& Summers, 1991; Attanasio \& Browning, 1995; Blundell, Browning \& Meghir, 1994; Attanasio \& Banks, 
1998). Razones vinculadas al riesgo de mortalidad, problemas de salud, herencias o restricciones de acceso al mercado de capital que dificulte e impida la realización de planes intertemporales justifican la coexistencia, en el inicio de la edad adulta, de procesos de acumulación de activos líquidos y desacumulación de activos ahorrados en el pasado (Butelmann \& Gallego, 2001).

En relación con el impacto sobre el tipo de interés, la teoría del ciclo vital postula un tipo de equilibrio inferior ante un escenario demográfico en el que aumenta el peso de los grupos de edad adulta Un aumento de la población adulta supondría un mayor volumen de ahorro que en condiciones ceteris paribus presionaría el tipo de interés a la baja. Al contrario, el acceso a la edad de jubilación de un volumen de población relevante contribuiría a la disminución del ahorro agregado presionando al alza el tipo de interés de equilibrio.

En la modelización del precio de los activos financieros normalmente el foco demográfico no se vislumbra y se centra en factores de coyuntura económica, toma de decisión de la autoridad monetaria, dinámica empresarial o acontecimientos políticos, fundamentalmente (Suárez-Lledó, 2015). No obstante, la hipótesis del ciclo de vital señala directamente el envejecimiento demográfico como factor determinante del nivel de ahorro agregado (Ando \& Modigliani, 1963; Modigliani, 1986). La tasa de descuento intertemporal podría depender de factores demográficos y a través de dicha vía influir en las decisiones de consumo y ahorro (Attanasio \& Browning, 1995; Baxter \& Jermann, 1999). La dinámica demográfica podría condicionar aspectos fundamentales del proceso de valoración de activos como es el tipo de interés real libre de riesgo y las primas de riesgo (Carroll \& Summers, 1991). La acumulación de riqueza y capital acometida por los adultos en previsión de sus necesidades de consumo en la vejez podría favorecer la productividad del factor trabajo y en consecuencia acelerar el crecimiento económico (Lee \& Mason, 2006).

La actitud del individuo al riesgo no es la misma en las diferentes etapas de su ciclo de vida y, en consecuencia, también las decisiones financieras a adoptar. La tolerancia al riesgo inherente a la bolsa es alta en la juventud e inicio de la edad adulta y baja en los tramos de edad próximos a la jubilación. En consecuencia, la composición de la cartera de activos además de las necesidades y posibilidades financieras se modifica siendo el peso de las acciones alto en el inicio y bajo al final. Desde dicha perspectiva, un escenario inversor con un marco demográfico envejecido favorecería una reducción de la renta variable, corriente vendedora, y consecuentemente las cotizaciones a la baja. En este sentido, algunos estudios atribuyen el auge bursátil sostenido del mercado de activos americano en los años ochenta y noventa al acceso a la cohorte de edad de 35 a 54 años de la generación al acceso a la edad media de los denominados baby boomers, nacidos durante el período de intensa natalidad tras la Segunda Guerra Mundial (Ostergaard, Sorensen \& Yosha 2002).

Algunos factores podrían mitigar los efectos del cambio demográfico, dado que las tendencias demográficas son predecibles y los agentes racionales deberían prever su impacto en la demanda de activos. La dinámica demográfica y económica actúan en horizontes temporales diferentes. Mientras que los cambios demográficos se perciben en el largo plazo, es a corto plazo cuando la actividad económica muestra su imagen. Ello significa que la articulación de cualquier medida o actuación de política demográfica o económica surtirá efectos en momentos muy diferentes del tiempo. Desde dicha perspectiva, el precio de los activos debería reflejar los efectos anticipados de los cambios demográficos. 
El envejecimiento poblacional está presente en la dinámica demográfica española, no solo porque los mayores viven más años, sino también porque hay menos jóvenes. La esperanza de vida, 83,09 años en 2017, se sitúa entre las más elevadas del entorno socioeconómico. La evolución del número de hijos por mujer constata el deterioro e implantación de un régimen de baja fecundidad, con una trayectoria decreciente hasta 1996 (1,16 hijos por mujer) que, a partir de 1999, se muestra fluctuante registrando en 2017 1,31 hijos por mujer. De forma preocupante se detecta, por primera vez, el descenso de la cohorte en edad de procrear, cuyos efectos no parecen estar suficientemente valorados (Redondo, Díaz-Fernández, Llorente-Marrón, Garay, Guidotti, \& Mendoza, 2015). La proyección demográfica del Instituto Nacional de Estadística (INE), en la misma línea, para un horizonte temporal de cincuenta años estima el aumento del peso de la cohorte de población de 65 y más años de edad que pasaría del 18,7 por ciento en 2016 al 24,9 en 2029 y 38,7 en 2064, respectivamente. En el mismo escenario, la proyección de la tasa de dependencia se incrementaría al 59,2 por ciento en 2029 y 95,6 por ciento en 2064.

En relación con la edad, la dinámica de los hogares españoles satisface los postulados de la teoría del ciclo vital. El gasto es creciente hasta la cohorte de 45 a 64 años, techo de la distribución a partir de la cual se inicia una ralentización y caída del consumo. Desde una perspectiva macroeconómica, el análisis teórico de las pautas de consumo de la economía española se centra fundamentalmente en los determinantes del ahorro familiar en función del origen de la renta, cambios de tenencia de los activos y riqueza de los hogares (Bover, 2008; Ferruz, Gómez \& López, 2012).

Según la Encuesta de Presupuestos Familiares del INE en 2007, el gasto medio de la categoría modal de la distribución gasto medio por hogar, cohorte de 45 a 64 años, que representa el 35,79 por ciento del total, alcanzó los 37.700,77 euros y en 2015 fue casi siete mil euros, inferior, aunque su peso aumentó hasta el 38,29 por ciento. El gasto de la cohorte de 65 y más años materializó un gasto inferior, que en 2007 alcanzó el 26,26 por ciento del total y en 2015 el 28,97, respectivamente.

El papel que las finanzas personales ocupan en el ciclo de vida del individuo y su conexión con el contexto demográfico no arroja muchas dudas, aunque no sea siempre claramente reconocido. Mediante el uso de técnicas econométricas aplicables a series temporales, el objetivo de este trabajo consiste en aproximar empíricamente dicha conexión al caso español, determinando un modelo de comportamiento dinámico entre el ciclo financiero, variable RATIO_PER y demográfico RATIO_DEMOGRAFICO, respectivamente.

\section{METODOLOGÍA}

En el ámbito de la investigación económica, el uso del análisis de cointegración reconcilia posturas aparentemente divergentes. La metodología econométrica se fundamenta en el conocimiento de la realidad económica y en la capacidad de establecimiento de relaciones causales. El análisis de cointegración, siempre que las relaciones fundamentales entre las variables económicas estén correctamente especificadas y respondan a un interés real de mejora del conocimiento económico, constituye un perfeccionamiento de la cuestión (Guisán, 2002).

El concepto de cointegración surge por el problema de intentar saber si dos o más variables están relacionadas y mantienen dicha relación a lo largo del tiempo. Que dos variables estén cointegradas implica que, aunque crezcan a lo largo del tiempo, lo hacen de forma sincronizada. El uso de las 
técnicas derivadas del análisis de cointegración permite obtener una relación de equilibrio a largo plazo y resultados libres de correlaciones espúreas y parametrizaciones inestables (Díaz-Fernández, Llorente-Marrón, \& Méndez-Rodríguez, 2018).

Cuando existe una combinación lineal estacionaria de orden cero, $I(0)$, de las variables $\boldsymbol{x}_{\boldsymbol{t}}$ e $\boldsymbol{y}_{\boldsymbol{t}}$, ambas integradas de primer orden, $I(1)$, diremos que están cointegradas. Ello implica la existencia de una relación de equilibrio a largo plazo. La cointegración entre dos variables $I(1)$ equivale a analizar la estacionariedad del término de perturbación aleatoria del modelo que las relaciona.

Los sistemas que, en su especificación incorporan variables cointegradas, de acuerdo con el teorema de representación de Granger, pueden ser formulados como MCE que permiten modelizar las relaciones de largo y corto plazo

$$
\Delta y_{t}=\gamma\left(\Delta x_{t}\right)+\alpha\left(y_{t-1}-\beta_{1}-\beta_{2} x_{t-1}\right)+\varepsilon_{t}
$$

donde, $\boldsymbol{\alpha}\left(\boldsymbol{y}_{\boldsymbol{t}-1}-\boldsymbol{\beta}_{1}-\boldsymbol{\beta}_{2} \boldsymbol{x}_{\boldsymbol{t}-1}\right)$ denota el MCE siendo $\boldsymbol{\alpha}<0$; el parámetro $\boldsymbol{\gamma}$ mide el efecto a corto plazo que la variable $\boldsymbol{x}_{\boldsymbol{t}}$ ejerce sobre $\boldsymbol{y}_{\boldsymbol{t}} ; \boldsymbol{\beta}_{2}$ recoge el efecto a largo plazo que la variable $\boldsymbol{x}_{\boldsymbol{t}}$ ejerce sobre $\boldsymbol{y}_{\boldsymbol{t}}$ y $\boldsymbol{\varepsilon}_{\boldsymbol{t}}$ el término de perturbación aleatoria, respectivamente. La formulación estacionaria de variables no estacionarias describe la variación de la variable $y_{t}$ alrededor de su tendencia a largo plazo en términos de factores exógenos, $x_{t}$, integrados de orden cero y la corrección del error $\boldsymbol{\alpha} \boldsymbol{u}_{t-1}=\boldsymbol{\alpha}\left(\boldsymbol{y}_{\boldsymbol{t}-1}-\boldsymbol{\beta}_{1}-\boldsymbol{\beta}_{2} \boldsymbol{x}_{\boldsymbol{t}-1}\right)$, error del equilibrio en el modelo de cointegración (Engle \& Granger, 1987).

Los pasos a seguir en el proceso se inician con el análisis de la estacionariedad de cada una de las series para evitar relaciones espúreas, la determinación de posibles relaciones de cointegración mediante el enfoque de máxima verosimilitud de Johansen (1988 y 1992) y la estimación, por último, del proceso dinámico de ajuste a la relación de equilibrio a largo plazo mediante un MCE.

En el caso que se ocupa, una aproximación a la conexión existente entre activo financiero y contexto demográfico se realiza desarrollando un ejercicio empírico que intenta capturar las relaciones temporales causales entre el indicador financiero y demográfico, respectivamente. Contrastar la existencia, o no, de una relación de equilibrio a largo plazo entre ambas variables, el carácter de exogeneidad o endogeneidad de los procesos dentro de la relación de equilibrio a largo plazo y el análisis de la interacción dinámica serán los pasos a seguir.

\section{RESULTADOS}

\section{Análisis empírico: el modelo y los datos}

Con objeto de analizar en qué medida la valoración de los activos financieros (RATIO_PER) y los cambios en la estructura por edades de la población (RATIO_DEMOGRAFICO) constituyen variables relacionadas entre sí a lo largo del tiempo, se evalúa empíricamente en España la modelización de la 
interrelación entre ambas series mediante la estimación de un MCE. La variable financiera, RATIO_PER recoge la relación entre el precio del activo financiero y el beneficio generado. En general, un valor alto denota elevadas expectativas de crecimiento por parte del mercado y por el contrario, un valor bajo un ritmo de crecimiento lento. La variable demográfica, RATIO_DEMOGRAFICO, se define como el cociente entre la población de edad mediana, cohorte de 45 a 49 años y la población de edad alrededor de la jubilación, cohorte de 60 a 64 años. Expresa la capacidad de renovación indicando cuántos efectivos poblacionales de la cohorte de 45 a 49 años hay por cada efectivo poblacional que accede a la denominada población adulta en un momento determinado. Dicha ratio se considera especialmente apropiado al comparar las dos cohortes de edad más significativas para decantar la balanza. Los jóvenes con carteras de acciones de escasa relevancia y los consolidados en la etapa de jubilación quedan excluidos del indicador. Un incremento de la ratio se asocia con un aumento en la valoración del activo financiero. La evolución de las variables RATIO_PER y RATIO_DEMOGRAFICO se recoge en la Figura 1. El período de análisis contiene cambios relevantes, la cohorte de 45 a 49 años de edad, población adulta de edad mediana superó los tres millones setecientos mil efectivos poblacionales, lo que supone un aumento del 69,73 por ciento y un millón menos la cohorte de 60 a 64 años, esto es, un aumento del 79,52 por ciento. Ambas cohortes sitúan temporalmente el inicio de su trayectoria vital en momentos diferentes del calendario. El aumento de la natalidad registrado en la segunda mitad del siglo XX, además de los flujos de inmigración exterior que paliaron los déficits de población, consecuencia de las generaciones vacías nacidas después de 1975 explican fundamentalmente la dinámica de la cohorte de edad mediana, grupo de edad de 45 a 49 años. El origen de la línea de vida correspondiente a la cohorte de 60 a 64 años de edad, se sitúa en el entorno de la tercera década del siglo XX.

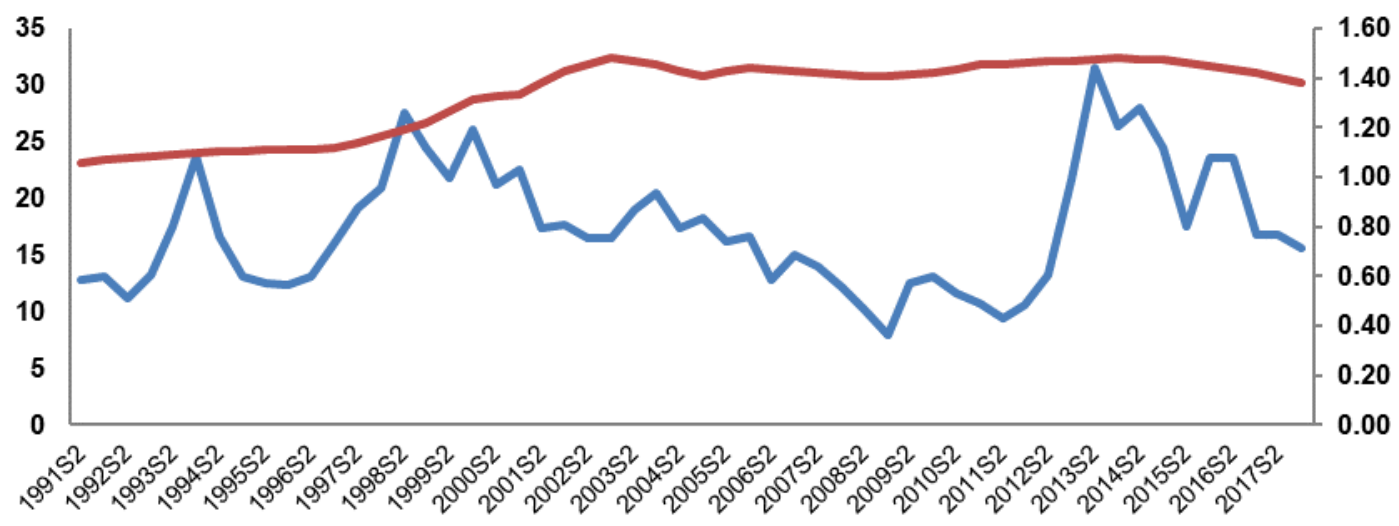

Ratio_PER Ratio_DEMOGRAFICO

Figura 1. Evolución temporal de las variables RATIO_PER y RATIO_DEMOGRAFICO

Figure 1. Evolution of the variables RATIO_PER and RATIO_DEMOGRAFICO over time. Fuente: elaboración propia con datos del Banco de España (2017).

En el mismo periodo, el sistema financiero registró una trayectoria expansiva y tamaño sobredimensionado que, a partir del inicio de la crisis económica, acusó fundamentalmente a través de la ruptura de los mecanismos de crédito. La actividad económica española tras una etapa de expansión en 2007 inició un proceso de desaceleración que concluyó dando lugar a un gran ajuste partir de 2008. 
Las propiedades estadísticas de la información muestral condicionan el enfoque metodológico a seguir. En este ejercicio se han tomado los datos de la serie Cifras de Población del INE y PER total Bolsa de Madrid del Banco de España, respectivamente. El periodo muestral, 54 observaciones de frecuencia semestral recogen las observaciones comprendidas entre el segundo semestre de 1991 y primer semestre de 2018. La evolución temporal de las series refleja, a priori, un comportamiento no estacionario.

Los modelos econométricos que utilizan series temporales ofrecen la posibilidad de verificar relaciones de largo plazo entre algunas variables de naturaleza económica. Dependiendo de las propiedades estadísticas de la información que se utilice, recogidas en la Tabla 1, la estimación del modelo dinámico que visualice la interrelación entre las variables seleccionadas puede ser realizada para el contraste.

Tabla 1. Estadísticos Básicos

Table 1. Basic statistics

\begin{tabular}{lcc}
\hline & RATIO_PER & RATIO_DEMOGRAFICO \\
\hline Media & 17.3067 & 1.3549 \\
\hline Mediana & 16.6600 & 1.3582 \\
\hline Desviación típica & 5.3949 & 0.2651 \\
\hline Asimetría & 0.5776 & 0.4470 \\
\hline Curtosis & 2.6282 & 2.0231 \\
\hline Jarque-Bera & 3.3142 & 3.9455 \\
\hline$p$-valor & 0.1906 & 0.1391 \\
\hline Correlación & & -0.2517 \\
\hline Observaciones & \multicolumn{2}{c}{54} \\
\hline \multicolumn{2}{l}{} \\
\end{tabular}

\section{Análisis de resultados}

Formalmente, se contrasta la hipótesis de estacionariedad siguiendo las pautas tradicionales de los estudios con series temporales. Mediante el test de Dickey-Fuller Aumentadas (ADF) y PhillipsPerron, respectivamente, se contrasta como hipótesis nula la existencia de raíces unitarias, comprobando si no es rechazada en niveles si lo es en primeras diferencias. Ambas series, RATIO_PER y RATIO_DEMOGRAFICO son integradas de orden uno, Tabla 2, y cointegradas (Tabla 3). Mediante el test de Johansen, según la prueba de la traza y del máximo valor propio para un nivel de significatividad del uno por ciento, se detecta la existencia de una relación de cointegración. Teniendo en cuenta que, de acuerdo con el teorema de representación de Granger, los sistemas que incorporan en su especificación variables cointegradas son normalmente formulados como Modelos de Corrección de Error (MCE).

$$
\begin{aligned}
& \Delta\left(\text { RATIO }_{-} P E R\right)=\alpha_{1}\left(\beta_{10}+\beta_{11} R A T I O_{-} P E R_{1-1}+\beta_{12} R A T I O_{-} D E M O G R A F I C O_{t-1}\right) \\
& +\sum_{i=1}^{p} \alpha_{1 i} \Delta(\text { RATIO_PER })_{t-i}+\sum_{i=1}^{p} \delta_{1 i} \Delta(\text { RATIO_DEMOGRAFICO })_{t-i}+\gamma Z_{t}+\varepsilon_{1 t} \\
& \Delta(\text { RATIO_DEMOGRAFICO })=\alpha_{2}\left(\beta_{20}+\beta_{21} \text { RATIO_PER } R_{1-1}+\beta_{22} \text { RATIO_DEMOGRAFICO }{ }_{t-1}\right) \\
& +\sum_{i=1}^{p} \alpha_{2 i} \Delta(\text { RATIO_PER })_{t-i}+\sum_{i=1}^{p} \delta_{2 i} \Delta(\text { RATIO_DEMOGRAFICO })_{t-i}+\gamma Z_{t}+\varepsilon_{2 t}
\end{aligned}
$$


Será el modelo a estimar denotando $\Delta\left(\boldsymbol{R A T I O} \boldsymbol{O}_{-} \boldsymbol{P E R}\right)$ y $\Delta\left(\boldsymbol{R A T I O _ { - }} \boldsymbol{D E M O G R A F I C O}\right)$ las variables endógenas del modelo en diferencias; $\boldsymbol{\alpha}_{i}$, la velocidad de ajuste a corto plazo para ambos ciclos, $\boldsymbol{\beta}$ y $\gamma$ los coeficientes a estimar correspondientes a valores pasados de las variables endógenas en diferencias y variables exógenas, respectivamente; y, $\boldsymbol{\varepsilon}$, el término de perturbación aleatoria distribuido de forma serialmente independiente.

Tabla 2. Test de raíces unitarias. 1991.S2-2018.S1

Table 2. Unit root test. 1991.S2-2018.S1

\begin{tabular}{|c|c|c|c|c|}
\hline \multicolumn{5}{|c|}{ Test de raíces unitarias } \\
\hline \multicolumn{5}{|c|}{ Augmented Dickey-Fuller test statistic (ADF) } \\
\hline Variable & Niveles & $p$-valor & Primeras diferencias & p-valor \\
\hline RATIO_PER & -0.650706 & 0.4306 & -7.242978 & 0.0000 \\
\hline RATIO_DEMOGRAFICO & 0.269254 & 0.7603 & -5.123106 & 0.0001 \\
\hline \multicolumn{5}{|c|}{ Phillips-Perron test statistic } \\
\hline Variable & Niveles & p-valor & Primeras diferencias & $p$-valor \\
\hline RATIO_PER & -0.650706 & 0.4306 & -7.242983 & 0.0000 \\
\hline RATIO_DEMOGRAFICO & 0.329440 & 0.7770 & -5.127201 & 0.0000 \\
\hline
\end{tabular}

Tabla 3. Test de raíces unitarias. 1991.S2-2018.S1

Table 3. Unit root test. 1991.S2-2018.S1

\begin{tabular}{lcccc}
\hline \multicolumn{4}{c}{ Test de cointegración de Johansen } \\
\hline & Trend assumption: No deterministic trend (restricted constant) & \\
\hline $\begin{array}{l}\text { Hypothesized } \\
\text { No. of CE(s) }\end{array}$ & Eigenvalue & Trace Statistic & 0.05 Critical Value & Prob.** $^{* *}$ \\
\hline None * & 0.295454 & 20.54908 & 15.49471 & 0.0079 \\
\hline At most 1 & 0.051356 & 2.688782 & 3.841466 & 0.1011 \\
\hline & $*$ denotes rejection of the hypothesis at the 0.05 level & \\
& \multicolumn{4}{c}{ Fuente: elaboración propia. }
\end{tabular}

Los resultados de la estimación se recogen en la Tabla 4. La regresión que aproxima la modelización del RATIO_PER es estadísticamente significativa de acuerdo con la prueba F-Snedecor, para un nivel de confianza del 99 por ciento y el coeficiente de determinación ajustado $\bar{R}^{2}=0,3057$. Se comprueba que los residuos satisfacen la hipótesis de independencia serial según el test de Portmanteau, homoscedasticidad y normalidad de acuerdo con la prueba de White y Jarque-Bera, respectivamente.

Teóricamente, el MCE captura tanto la relación estable a largo plazo con efectos permanentes como la de corto plazo con efectos transitorios. La relación de largo plazo se interpreta como el reflejo de una situación en la que cada unidad de activo financiero, RATIO_PER, se asocia con un registro menor de la variable demográfica, RATIO_DEMOGRAFICO mostrando un modelo persistente y estable durante el período analizado. La estimación obtenida de la ecuación de cointegración visualiza el dominio y significatividad estadística del factor demográfico a largo plazo, en relación con el corto plazo en la determinación de la trayectoria del ciclo financiero. Se contrasta la significatividad estadística y dominio del efecto del factor demográfico a largo plazo $\left[\hat{\boldsymbol{\beta}}_{12}=-8.075624\left(\boldsymbol{t}^{*}=-2.11852\right)\right]$ en relación con el corto plazo $\left[\hat{\boldsymbol{\delta}}_{12}=-9.424834(\boldsymbol{t} *=-1.29648)\right]$. El 
MCE captura esta relación a la vez que distingue entre la causalidad del efecto de sustitución procedente de la relación con efectos permanentes (largo plazo) y transitorios (corto plazo). El modelo no captura el efecto transitorio, dado que no se obtienen parámetros estadísticamente significativos en la corrección de error (corto plazo) para ninguno de los ciclos analizado.

El término de corrección de error (o parámetro de convergencia) del ciclo financiero [CointEq1:-0.355242 $(\boldsymbol{p}$-valor $<0.001)]$ y demográfico $\quad[$ CointEq1: $-0.003064(\boldsymbol{p}$-valor $<0.05)]$ refleja el efecto sistemático de sustitución en el largo plazo, estadísticamente significativo en ambos casos. Ello significa que la relación de largo plazo contribuye a la restauración de la relación de equilibrio de las series cuando se produce una perturbación inesperada en el corto plazo. El modelo parece adelantar un efecto de equilibrio a través de la sustitución en el que un aumento en el ciclo demográfico implicaría una reducción inmediata en el financiero. Las variables dummy incluidas con especificación multiplicativa entre los años $2013[0.384456(\boldsymbol{p}$-valor $<0.001)]$ y 2016 $[0.201514(\boldsymbol{p}-$ valor $<0.05)]$ justifican cambios estructurales en la dinámica del ciclo financiero.

Tabla 4. Estimación modelo de corrección de error

Table 4. Estimation of the error correction model

\begin{tabular}{|c|c|c|c|}
\hline \multicolumn{2}{|c|}{ Vector Error Correction Estimates } & \multicolumn{2}{|c|}{ Included observations: 52} \\
\hline \multicolumn{2}{|c|}{ CointegratingEq: Largo plazo } & \multicolumn{2}{|c|}{ CointEq1 } \\
\hline \multicolumn{2}{|c|}{ RATIO_PER(-1) } & \multicolumn{2}{|c|}{1.0000} \\
\hline \multicolumn{2}{|c|}{ RATIO_DEMOGRAFICO(-1) } & \multicolumn{2}{|c|}{$8.075624 * * *$} \\
\hline \multicolumn{2}{|c|}{$\mathrm{C}$} & \multicolumn{2}{|c|}{-28.39759} \\
\hline \multicolumn{2}{|c|}{ Error Correction: } & D(RATIO_PER) & D(RATIO_DEMOGRAFICO) \\
\hline \multicolumn{2}{|c|}{ CointEq1 } & $-0.355242 * * *$ & $-0.003064 * *$ \\
\hline \multicolumn{2}{|c|}{ RATIO_PER(-1) } & 0.007146 & 0.001096 \\
\hline \multicolumn{2}{|c|}{ RATIO_DEMOGRAFICO(-1) } & -9.424834 & 0.220734 \\
\hline \multicolumn{2}{|c|}{ C } & -0.289529 & 0.006354 \\
\hline \multicolumn{2}{|c|}{ D2013*RATIO_PER } & $0.384456 * * *$ & -0.001599 \\
\hline \multicolumn{2}{|c|}{ D2016*RATIO_PER } & $0.201514^{* *}$ & $5.97 \mathrm{E}-05$ \\
\hline \multicolumn{2}{|c|}{$\mathrm{R}^{2}$} & 0.373778 & 0.159070 \\
\hline \multicolumn{2}{|c|}{$\mathrm{R}^{2}$-ajustado } & 0.305710 & 0.067665 \\
\hline \multicolumn{2}{|c|}{ Estadistico F-Snedecor } & $5.491266 * * *$ & 1.740271 \\
\hline \multirow{6}{*}{ Residuos } & \multirow{2}{*}{ Test normalidad } & Jarque-Bera & $p$-valor \\
\hline & & 1.1852 & 0.5528 \\
\hline & \multirow{2}{*}{ Test correlación } & LM orden 2 & p-valor \\
\hline & & 5.693160 & 0.2233 \\
\hline & \multirow{2}{*}{ Test heteroscedasticidad } & White & $p$-valor \\
\hline & & 16.84458 & 0.8552 \\
\hline
\end{tabular}

El comportamiento de la Función de Impulso- Respuesta (FIR) recoge la reacción de las variables del sistema ante shocks no anticipados en los errores visualizando el efecto en tiempo, dirección e intensidad. En la Figura 2, se visualiza la respuesta de la serie financiera a alteraciones en la misma como un escenario de trayectoria decreciente permanente en el tiempo. Cuando los cambios se producen en el factor demográfico, la respuesta de la serie financiera nula inicialmente pasa a tener signo negativo y magnitud estable a partir del segundo período posterior al shock. La correlación 
inversamente proporcional entre ambos ciclos se visualiza también al analizar la respuesta del factor demográfico ante alteraciones en el comportamiento de la variable financiera.

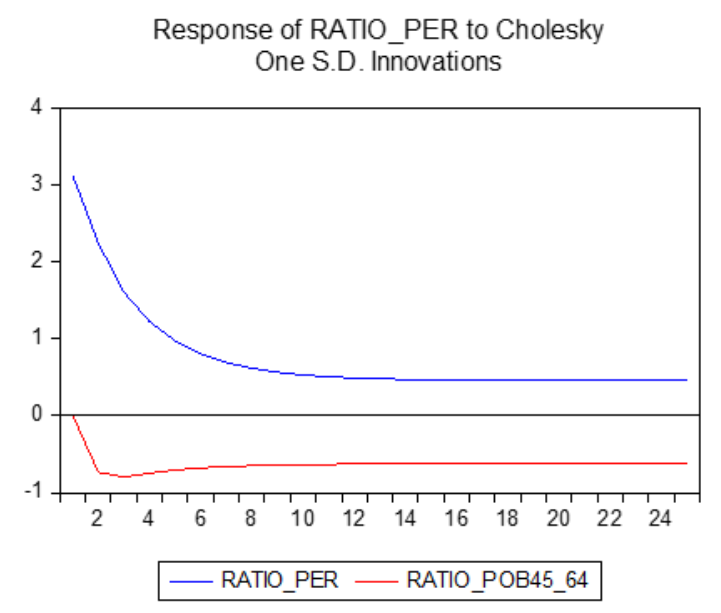

Response of RATIO_POB45_64 to Cholesky

One S.D. Innovations

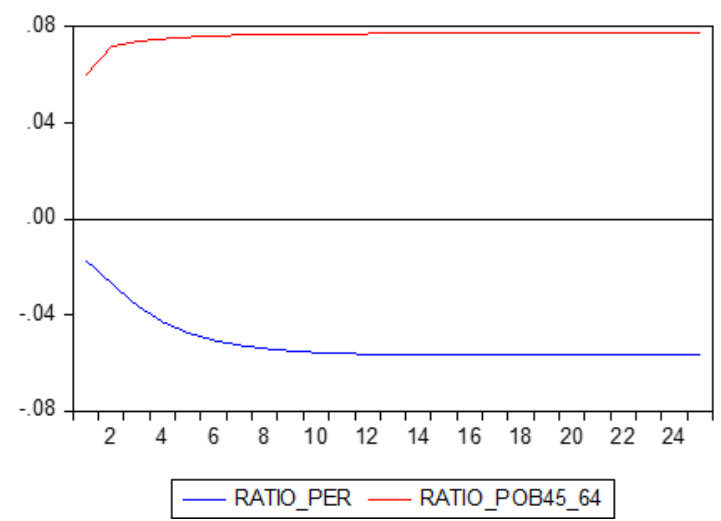

Figura 2. Función impulso-respuesta

Figure 2. Impulse-response function

Fuente: elaboración propia.

La vinculación entre ambos ciclos se refrenda igualmente en el análisis de la descomposición de la varianza, recogido en la Tabla 5, que profundiza los resultados al visualizar el peso de cada variable en la determinación de la desviación típica del error de predicción. El error de predicción de RATIO_PER después de dos años (4 semestres) del shock viene explicado principalmente por sí mismo, 91,59 por ciento, el RATIO_DEMOGRAFICO representa el 8,41 por ciento. Cuatro años (8 semestres) después del shock su peso disminuye hasta el 85,74 por ciento y el peso del RATIO_DEMOGRAFICO aumenta 5,85 puntos porcentuales (14,26 por ciento). Pasados diez (20 semestres) y doce (24 semestres) años del shock el aumento del peso del factor demográfico es claramente significativo, 18,67 y 25,48 por ciento, respectivamente. En el mismo periodo se observa también la relación inversa, esto es, el peso que en el error de predicción del factor demográfico desempeña el factor financiero. La asociación inversamente proporcional se aprecia en la correlación existente entre ambas series $(-0,2517)$. 
Tabla 5. Análisis de descomposición de la varianza

Table 5. Variance decomposition analysis

\begin{tabular}{lccc}
\hline \multirow{2}{*}{$\begin{array}{l}\text { Porcentaje del error de } \\
\text { predicción }\end{array}$} & Semestre & \multicolumn{2}{c}{ Explicado por } \\
\cline { 2 - 4 } & & RATIO_PER & RATIO_DEMOGRAFICO \\
\cline { 2 - 4 } & 4 & 91.59079 & 8.409210 \\
\cline { 2 - 4 } RATIO_PER & 12 & 85.73873 & 14.26127 \\
\cline { 2 - 4 } & 20 & 81.32146 & 18.67854 \\
\cline { 2 - 4 } & 24 & 74.51787 & 25.48213 \\
\hline \multirow{3}{*}{ RATIO_DEMOGRAFICO } & 4 & 71.81850 & 82.59740 \\
\cline { 2 - 4 } & 12 & 25.45940 & 74.54060 \\
\cline { 2 - 4 } & 20 & 28.92958 & 71.07042 \\
\cline { 2 - 4 } & 24 & 31.68324 & 68.31676 \\
\hline
\end{tabular}

\section{CONCLUSIONES}

El papel que las finanzas personales ocupan en el ciclo de vida del individuo y su conexión con el contexto demográfico es indudable, aunque no sea siempre claramente reconocido. En su teoría del ciclo de vida, Modigliani (1986) contribuyó decisivamente a despertar en la sociedad la preocupación sobre el bienestar futuro, además de crear una cultura del ahorro.

El trabajo desarrolla un análisis de cointegración para explorar e identificar la relación dinámica existente entre activo financiero y contexto demográfico, en España. La hipótesis del ciclo de vida sugiere que los individuos planifican su consumo y comportamiento del ahorro a lo largo de su ciclo de vida. Se desea comprobar la vinculación existente entre el consumo de activos financieros y los cambios en la estructura de edad de la población, en concreto cuando los grupos de edad adulta ganan peso en relación al tamaño total de la población.

Se aplica un análisis de cointegración siguiendo la metodología de Johansen (1988) y Engle \& Granger (1987), que aproxima la interrelación existente entre el indicador financiero y demográfico. A partir de información estadística correspondiente a la dinámica de las variables RATIO_PER, Y RATIO_DEMOGRAFICO se estima un MCE en España durante el periodo comprendido entre los años 1991 y 2018, con frecuencia semestral que aproxima la interacción y regularidades empíricas existentes (Sims, 1980).

Desde una perspectiva metodológica, el uso de técnicas derivadas del análisis de cointegración permite avanzar en el conocimiento y constituye un perfeccionamiento de la cuestión aproximando el conocimiento real del fenómeno económico en etapas avanzadas de su análisis (Guisán, 2002). La metodología econométrica se fundamenta en el conocimiento de la realidad económica y en la capacidad de establecimiento de relaciones causales. A este respecto destaca la oportunidad del trabajo desde una doble perspectiva. La relación analizada se corresponde con un enfoque analizado y recogido en la literatura económica con planteamientos y tratamientos diferentes desde una perspectiva temporal y territorial (Gillen \& Guccione, 1970; Selvanathan, 1991). 
La estimación realizada proporciona evidencia empírica de la asociación entre ambas variables. El indicador demográfico compara las dos cohortes más significativas en el análisis planteado excluyendo a los muy jóvenes por la escasa relevancia de sus carteras de acciones y a los que ya llevan tiempo en la jubilación. Un incremento de esta ratio se asocia con un aumento de la valoración de las acciones. La relación a estimar se modeliza utilizando un MCE. El análisis realizado revela que la relación existente entre RATIO_PER y RATIO_DEMOGRAFICO es permanente, de largo plazo e inversamente proporcional.

El análisis del envejecimiento, fenómeno determinado por la rigidez con la que se aborda el tránsito de las cohortes a partir de la barrera de los 65 años puede deparar sorpresas. Para las próximas décadas, un horizonte demográfico de claro envejecimiento no propicia un escenario de optimismo inversor. La reducción del ahorro presionara al alza el tipo de interés real libre de riesgo aumentando la prima bursátil como consecuencia de la mayor proporción de personas en plena edad de jubilación o entorno. Ello implicará una tasa de descuento más elevada que tenderá a presionar a la baja los precios de los activos. No obstante, no se debe olvidar que el proceso de recomposición de carteras por parte de la población jubilada es más lento que el de los trabajadores en edad de ahorrar, por lo que el efecto negativo sobre la renta variable podría ser más tenue.

\section{REFERENCIAS}

Ando, A., \& Modigliani, F. (1963). The life cycle hypothesis of saving: Aggregate implications and tests. The American Economic Review, 53(1), 55-84. Recuperado de www.jstor.org/stable/1817129

Attanasio, O., \& Banks, J. (1998). Trends in household saving: a tale of two countries, IFS Working Paper series, W98/15. Recuperado de https://www.ifs.org.uk/publications/2729

Attanasio, O. P., \& Browning, M. (1995). Consumption over the life cycle and over the business cycle. American Economic Review, 85(5), 1118-1137. Recuperado de https://www.jstor.org/stable/2950978

Banco de España (2017). Cotización y contratación. Recuperado de https://www.bde.es/webbde/es/estadis/infoest/temas/sb cotiz.html

Baxter, M., \& Jermann, U. (1999). Household Production and the Excess Sensitivity of Consumption to Current Income. American Economic Review, 89(4), 902-920. https://doi.org/10.1257/aer.89.4.902

Blundell, R., Browning, M. \& Meghir, C. (1994). Consumer Demand and the Life-Cycle Allocation of Household Expenditures. Review of Economic Studies, 61(1), 57-80. https://doi.org/10.2307/2297877

Bover, O. (2008). Dinámica de la renta y la riqueza de las familias españolas: resultados del panel de La Encuesta Financiera de las Familias (EFF) 2002-2005. Documentos ocasionales - Banco de España, (10), 1-35. Recuperado de 
https://www.bde.es/f/webbde/SES/Secciones/Publicaciones/PublicacionesSeriadas/Docume ntosOcasionales/08/Fic/do0810.pdf

Butelmann, A., \& Gallego, F. (2001). Household Saving in Chile: Microeconomic Evidence. Recuperado de https://www.bcentral.cl/web/guest/-/household-saving-in-chile-microeconomic-eviden-2

Cabré i Pla, A., \& Pérez Díaz, J. (1996). Envejecimiento demográfico en España. En Las actividades económicas de las personas mayores. Madrid: Central Hispano, pp. 33-60. Recuperado de http://hdl.handle.net/10261/38745

Carroll, C.D., \& Summers, L.H. (1991). Consumption growth parallels income growth: some new evidence. En B. Douglas Bernheim and John B. Shoven (Ed.), National Saving and Economic Performance (pp. 305-347). Recuperado de https://ideas.repec.org/h/nbr/nberch/5995.html

Deaton, A. (1997). The Analysis of Household Surveys: A Microeconometric Approach to Development Policy Baltimore MD: Johns Hopkins University Press (Published for the World Bank). American Journal of Agricultural Economics, 82(3), 780-782. https://doi.org/10.1093/ajae/82.3.780

Díaz-Fernández, M. (2018). Envejecimiento y finanzas. Tiempo de paz, 130, 124-129. Recuperado de https://bit.ly/30deAHT

Díaz-Fernández, M., Llorente-Marrón, M. \& Méndez-Rodríguez, P. (2018). Interrelation between births and the housing market: A cointegration analysis for the Spanish case. Population, Space and Place, 25(2), https://doi.org/10.1002/psp.2172

Engle, R. F. \& Granger, C. W. J. (1987). Co-Integration and Error Correction: Representation, Estimation, and Testing. Econometrica, 55(2), 251-276. https://doi.org/10.2307/1913236

Ferruz, L., Gómez, A. \& López, F. (2012). El ahorro de los hogares de España. Análisis Financiero, 120, 19-31. Recuperado de https://dialnet.unirioja.es/servlet/articulo?codigo=4537420

Gillen, W. J. \& Guccione, A. (1970). The Estimation of Postwar Regional Consumption Functions in Canada. The Canadian Journal of Economics, 3(2), 276-291. https://doi.org/10.2307/133677

Gourinchas, P. O. \& Parker, J. A. (2001). The Empirical Importance of Precautionary Saving. American Economic Review, 91(2). 406-412. https://doi.org/10.1257/aer.91.2.406

Guisán, C. (2002). Causalidad y cointegración en modelos econométricos: aplicaciones a los países de la OCDE y limitaciones de los test de cointegración. Series Economic Development Universidad de Santiago de Compostela, Working Paper 61. Recuperado de http://www.usc.es/economet/aeeadepdf/aeeade61.pdf

Johansen, S. (1992). Determination of cointegration rank in the presence of a linear trend. Oxford Bulletin of Economics and Statistics, 54(3), 383-398. https://doi.org/10.1111/j.14680084.1992.tb00008.x 
Johansen, S. (1988): Statistical analysis of cointegration vectors. Journal of Economics Dynamics and Control, 12(2-3), 231-254. https://doi.org/10.1016/0165-1889(88)90041-3

Lee, R. \& Mason, A. (2006). What is the Demographic Dividend? Finance \& Development, 43(3). Recuperado de https://bit.ly/307gbPq

Modigliani, F. (1986). Life cycle, individual thrift and the wealth of nations. Science, 234(4777), 704712. https://doi.org/10.1126/science.234.4777.704

Ostergaard, C., Sorensen B.E. \& Yosha, O. (2002). Consumption and Aggregate Contraints: Evidence from US States and Canadian Provinces. Journal of Political Economy, 110(3), 634-645. https://doi.org/10.1086/339724

Pinto, G. (2016). El bono demográfico en América Latina. El efecto económico de los cambios en la estructura por edad de una población. Población y Salud en Mesoamérica, 13(2), 1-17. http://dx.doi.org/10.15517/psm.v13i2.21863

Paxson, C. (1996). Saving and growth: Evidence from micro data. European Economic Review, 40(2), 255-288. https://doi.org/10.1016/0014-2921(95)00063-1

Pérez, J. (2005). Consecuencias sociales del envejecimiento demográfico. Papeles de Economía Española, 104, 210-226. Recuperado de https://bit.ly/32boqMk

Redondo, N., Díaz-Fernández, M., Llorente-Marrón, M., Garay, S., Guidotti, C. y Mendoza, L. (2015). El espacio residencial del cuidado de los adultos mayores en América Latina y España. Notas de Población, 100(1), 223-258. Recuperado de https://repositorio.cepal.org/handle/11362/38529

Roa García, M. J. (2008). Cambio demográfico y desarrollo financiero: efectos sobre el crecimiento económico. Revista Empresa y Humanismo, XI(1/08), 231-272. Recuperado de https://bit.ly/2LFtYsw

Selvanathan, S. (1991). Regional Consumption Patterns in Australia: A System-Wide Analysis. Economic Record, 67(4), 338-346. https://doi.org/10.1111/j.1475-4932.1991.tb02563.x

Sims, C. (1980). Macroeconomics and reality. Econometrica, 48(1), 1-48. https://doi.org/10.2307/1912017

Suárez-Lledó, J. (2015). Demografía y precios de los activos financieros: se agotan los vientos de cola. Informe Mensual. Recuperado de http://www.caixabankresearch.com/demografia-y-preciosde-los-activos-financieros-se-agotan-los-vientos-de-cola-d2 